Musées, Patrimoine et Culture scientifiques et techniques

$174 \mid 2017$

novembre-décembre 2017

\title{
Boutiques de musées : écueils et bonnes pratiques
}

\section{Laure Danilo}

\section{OpenEdition \\ Journals}

Édition électronique

URL : http://journals.openedition.org/ocim/1868

DOI : 10.4000/ocim. 1868

ISSN : 2108-646X

Éditeur

OCIM

Édition imprimée

Date de publication : 1 novembre 2017

Pagination : 11-17

ISSN : 0994-1908

Référence électronique

Laure Danilo, « Boutiques de musées : écueils et bonnes pratiques », La Lettre de I'OCIM [En ligne], 174 |

2017, mis en ligne le 01 novembre 2018, consulté le 21 novembre 2019. URL : http://

journals.openedition.org/ocim/1868; DOI : 10.4000/ocim.1868

Ce document a été généré automatiquement le 21 novembre 2019.

Tous droits réservés 


\title{
Boutiques de musées : écueils et bonnes pratiques
}

\author{
Laure Danilo
}

Librairie-Boutique du musée de l'Homme, gérée par la RMN-GP.

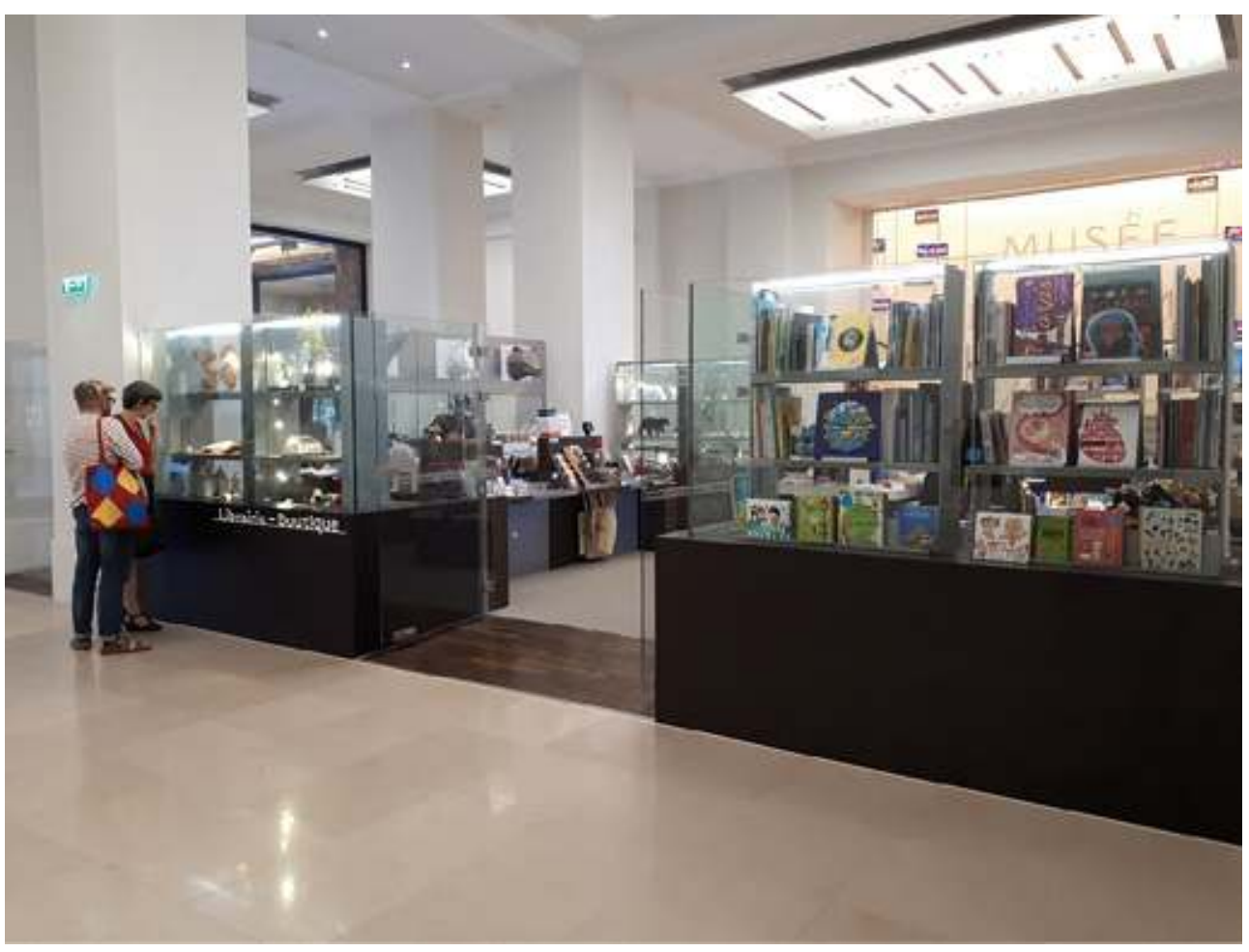

(c) L. Danilo

1 Les musées scientifiques, et notamment les muséums d'histoire naturelle, sont aujourd'hui peu nombreux à porter des projets de boutiques en leur sein. Pourtant, ces lieux, loin d'être de simples artifices commerciaux, doivent permettre au visiteur d'agrémenter et de prolonger sa visite et au musée de transmettre, d'une manière 
alternative, ses valeurs et son image. Les boutiques, comme d'autres services aux publics, font partie de ces espaces à développer dans une vision de musées qui ne sont pas uniquement des lieux d'exposition. La faible représentativité des muséums dans ce secteur peut être reliée à plusieurs éléments, dont une méconnaissance de ce domaine et des écueils à éviter, malgré quelques écrits sur le sujet (Deval, 1994 ; Bacher, 2000 ; Cam, 2008). En outre, la boutique est souvent perçue comme un poids financier, notamment si des postes lui sont dédiés, alors qu'elle peut être envisagée différemment, y compris dans un établissement de petite ou moyenne taille.

2 Pour palier ce manque, une enquête comparative de boutiques d'établissements culturels, en particulier scientifiques, a été réalisée en 2016 afin de mettre en exergue des bonnes pratiques et des écueils à éviter lors de la conception et de la gestion d'une boutique de musée. Sur 48 établissements contactés, 22 ont pu fournir des éléments détaillés, au cours d'un questionnaire administré lors d'entretiens téléphoniques.

\section{Une enquête comparative pour embrasser le sujet}

3 Afin d'évaluer les meilleures stratégies développées, l'analyse a porté sur différents aspects inhérents à la constitution et à la gestion d'une boutique. L'enquête comparative a pris la forme d'un questionnaire de 25 questions proposées au cours d'un entretien d'une vingtaine de minutes. Huit catégories sont explorées (données générales sur l'établissement, situation de la boutique, agents, produits, communication, gestion et finances). Les questions sont fermées ou à choix multiples, afin de favoriser le traitement statistique des résultats. Un champ libre à l'issue du questionnaire permet de recueillir des informations complémentaires.

4 Le temps annoncé de 20 minutes, a régulièrement été dépassé, les interlocuteurs se montrant fréquemment intéressés et communicatifs. Ce fut un avantage considérable, de nombreuses informations périphériques ayant été obtenues grâce à ces moments d'échange et de discussion.

\section{Un échantillon d'établissements culturels}

5 La constitution de l'échantillon a été réalisée suivant plusieurs critères. Le seuil de $20 \mathrm{~m}^{2}$ a été retenu pour définir une boutique, les surfaces inférieures constituant généralement de simples comptoirs de vente. L'échantillonnage s'est principalement axé sur des établissements de culture scientifique, soit les muséums d'histoire naturelle et les centres de sciences. Afin d'explorer et comparer ce qui se fait dans d'autres domaines, des musées d'art, d'histoire et de technique ont également été sollicités. Un détour par des structures plus orientées dans la vente tout en demeurant dans le champ culturel au sens large, tels qu'un office de tourisme et des aquariums permet de bénéficier de l'expertise de professionnels de la vente.

6 Au total, 48 établissements ont été contactés. Tous les muséums d'Histoire naturelle et les centres de science susceptibles de disposer d'une boutique ont été approchés, mais seuls respectivement huit et deux d'entre eux répondaient aux critères (boutique supérieure à $20 \mathrm{~m}^{2}$ ). Sur les six aquariums sollicités, trois n'ont pas donné suite. Il s'ensuit un échantillon de 22 établissements, dont plus de la moitié positionnés dans le domaine scientifique. 
Établissements ayant répondu à l'enquête comparative menée à l'automne 2016.

\begin{tabular}{|c|c|c|}
\hline Nom de l'établissement & Localisation & Type d'établissement \\
\hline Grande Galerie de l'Evolution & MNHN, Paris & Muséum d'histoire naturelle \\
\hline Muséum d'Histoire Naturelle de Bourges & Bourges & Muséum d'histoire naturelle \\
\hline Musée des Confluences & Lyon & Muséum d'histoire naturelle \\
\hline Muséum d'Histoire Naturelle de Grenoble & Grenoble & Muséum d'histoire naturelle \\
\hline Muséum d'Histoire Naturelle du Havre & Le Havre & Muséum d'histoire naturelle \\
\hline Muséum d'Histoire Naturelle de La Rochelle & La Rochelle & Muséum d'histoire naturelle \\
\hline \multicolumn{3}{|l|}{ Muséum National d'Histoire Naturelle du } \\
\hline Luxembourg & Luxembourg & Muséum d'histoire naturelle \\
\hline Muséum d'Histoire Naturelle de Toulouse & Toulouse & Muséum d'histoire naturelle \\
\hline Musée de l'Homme & MNHN, Paris & Musée scientifique \\
\hline \multicolumn{3}{|l|}{ Jardins Botaniques du Grand Nancy et de } \\
\hline I'Université de Lorraine & Villers-lès-Nancy & Etablissement de culture scientifique \\
\hline Cap Sciences & Bordeaux & Centre de sciences (CCSTI) \\
\hline Le Vaisseau & Strasbourg & Centre de sciences (CCSTI) \\
\hline Aquarium de La Rochelle & La Rochelle & Aquarium \\
\hline Cité de la Mer & Cherbourg & Aquarium \\
\hline Nausicaa & Boulogne-sur-Mer & Aquarium \\
\hline Musée du Temps & Besançon & Musée d'histoire et de technique \\
\hline Les Machines de l'ile & Nantes & Espace d'exposition technique et artistique \\
\hline Musée du Nouveau Monde & La Rochelle & Musée d'histoire et d'ethnologie \\
\hline Musée des Beaux-arts & Nancy & Musée de Beaux-arts \\
\hline Musée Fabre & Montpellier & Musée de Beaux-arts \\
\hline Musée Archéologique Saint Raymond & Toulouse & Musée archéologique \\
\hline Nancy Tourisme & Nancy & Office de tourisme \\
\hline
\end{tabular}




\begin{tabular}{|c|c|c|}
\hline Nom de l'établissement & Localisation & Type d'établissement \\
\hline Grande Galerie de I'Evolution & MNHN, Paris & Muséum d'histoire naturelle \\
\hline Muséum d'Histoire Naturelle de Bourges & Bourges & Muséum d'histoire naturelle \\
\hline Musée des Confluences & Lyon & Muséum d'histoire naturelle \\
\hline Muséum d'Histoire Naturelle de Grenoble & Grenoble & Muséum d'histoire naturelle \\
\hline Muséum d'Histoire Naturelle du Havre & Le Havre & Muséum d'histoire naturelle \\
\hline Muséum d'Histoire Naturelle de La Rochelle & La Rochelle & Muséum d'histoire naturelle \\
\hline \multicolumn{3}{|l|}{ Muséum National d'Histoire Naturelle du } \\
\hline Luxembourg & Luxembourg & Muséum d'histoire naturelle \\
\hline Muséum d'Histoire Naturelle de Toulouse & Toulouse & Muséum d'histoire naturelle \\
\hline Musée de l'Homme & MNHN, Paris & Musée scientifique \\
\hline \multicolumn{3}{|l|}{ Jardins Botaniques du Grand Nancy et de } \\
\hline I'Université de Lorraine & Villers-lès-Nancy & Etablissement de culture scientifique \\
\hline Cap Sciences & Bordeaux & Centre de sciences (CCSTI) \\
\hline Le Vaisseau & Strasbourg & Centre de sciences (CCSTI) \\
\hline Aquarium de La Rochelle & La Rochelle & Aquarium \\
\hline Cité de la Mer & Cherbourg & Aquarium \\
\hline Nausicaa & Boulogne-sur-Mer & Aquarium \\
\hline Musée du Temps & Besançon & Musée d'histoire et de technique \\
\hline Les Machines de l'lle & Nantes & Espace d'exposition technique et artistique \\
\hline Musée du Nouveau Monde & La Rochelle & Musée d'histoire ot d'ethnologie \\
\hline Musée des Beaux-arts & Nancy & Musée de Beaux-arts \\
\hline Musée Fabre & Montpellier & Musée de Beaux-arts \\
\hline Musée Archéologique Saint Raymond & Toulouse & Musée archéologique \\
\hline Nancy Tourisme & Nancy & Office de tourisme \\
\hline Nom de l'établissement & Localisation & Type d'établissement \\
\hline Grande Galerie de l'Evolution & MNHN, Paris & Muséum d'histoire naturelle \\
\hline Muséum d'Histoire Naturelle de Bourges & Bourges & Muséum d'histoire naturelle \\
\hline Musée des Confluences & Lyon & Muséum d'histoire naturelle \\
\hline Muséum d'Histoire Naturelle de Grenoble & Grenoble & Muséum d'histoire naturelle \\
\hline Muséum d'Histoire Naturelle du Havre & Le Havre & Muséum d'histoire naturelle \\
\hline Muséum d'Histoire Naturelle de La Rochelle & La Rochelle & Muséum d'histoire naturelle \\
\hline \multicolumn{3}{|l|}{ Muséum National d'Histoire Naturelle du } \\
\hline Luxembourg & Luxembourg & Muséum d'histoire naturelle \\
\hline Muséum d'Histoire Naturelle de Toulouse & Toulouse & Muséum d'histoire naturelle \\
\hline Musée de l'Homme & MNHN, Paris & Musée scientifique \\
\hline \multicolumn{3}{|l|}{ Jardins Botaniques du Grand Nancy et de } \\
\hline I'Université de Lorraine & Villers-lès-Nancy & Etablissement de culture scientifique \\
\hline Cap Sciences & Bordeaux & Centre de sciences (CCSTI) \\
\hline Le Vaisseau & Strasbourg & Centre de sciences (CCSTI) \\
\hline Aquarium de La Rochelle & La Rochelle & Aquarium \\
\hline Cité de la Mer & Cherbourg & Aquarium \\
\hline Nausicaa & Boulogne-sur-Mer & Aquarium \\
\hline Musée du Temps & Besançon & Musée d'histoire et de technique \\
\hline Les Machines de l'ile & Nantes & Espace d'exposition technique et artistique \\
\hline Musée du Nouveau Monde & La Rochelle & Musée d'histoire et d'ethnologie \\
\hline Musée des Beaux-arts & Nancy & Musée de Beaux-arts \\
\hline Musée Fabre & Montpellier & Musée de Beaux-arts \\
\hline Musée Archéologique Saint Raymond & Toulouse & Musée archéologique \\
\hline Nancy Tourisme & Nancy & Office de tourisme \\
\hline
\end{tabular}

Certaines enquêtes demeurent incomplètes, lorsque les établissements n'ont pas ou peu de regard analytique sur leur gestion de boutique, ce qui conduit à l'ignorance de certaines données pourtant essentielles pour évaluer la rentabilité de l'équipement ; ou lorsque les interlocuteurs ne souhaitent pas communiquer leurs données, en général dans le cas de structures privées ou de grands établissements publics. 


\section{Résultats quantitatifs : entre intuitions confirmées et inattendus dévoilés}

7 Si une boutique est plus qu'un espace de vente, cette étude s'attache à analyser la gestion d'une boutique d'un point de vue commercial. Cette approche vise à épauler d'éventuelles constitutions de boutiques, en évitant qu'elles ne deviennent un poids financier pour l'établissement, mais qu'au contraire elles puissent, par leurs recettes, contribuer à son effort financier. Ces boutiques ne peuvent certes être considérées comme des sources de revenus lorsque leurs chiffres d'affaires sont comparés aux coûts globaux d'un établissement, notamment du point de vue de la masse salariale. Cependant, l'apport symbolique d'une recette supplémentaire peut être un atout à valoriser auprès de sa tutelle, de la même manière que lorsqu'un petit mécénat est levé.

Aussi, cette étude cherche à identifier les éléments qui conduisent une boutique à mieux vendre ses produits, à travers plusieurs aspects : le personnel, les produits eux-mêmes, la communication... De premières analyses (régressions linéaires) ont cherché à déterminer ce qui pouvait influer sur le rapport ${ }^{1}$. Ces régressions linéaires ont montré certains liens entre le rapport et d'autres variables, mais la situation est plus complexe : un faisceau de variables interdépendantes influence le rapport. Afin de mieux les appréhender, une analyse multivariée (ACP) a alors été conduite. Un traitement qualitatif a ensuite été effectué. Il s'agissait de faire ressortir les éléments qui revenaient à plusieurs reprises dans les enquêtes. Certains recoupent des résultats des analyses statistiques, d'autres apportent des compléments d'information essentiels.

Un exemple de stratégie pour créer l'événement et stimuler les ventes : l'accueil personnalisé selon l'exposition temporaire en cours. Grande Galerie de l'Évolution, MNHN.

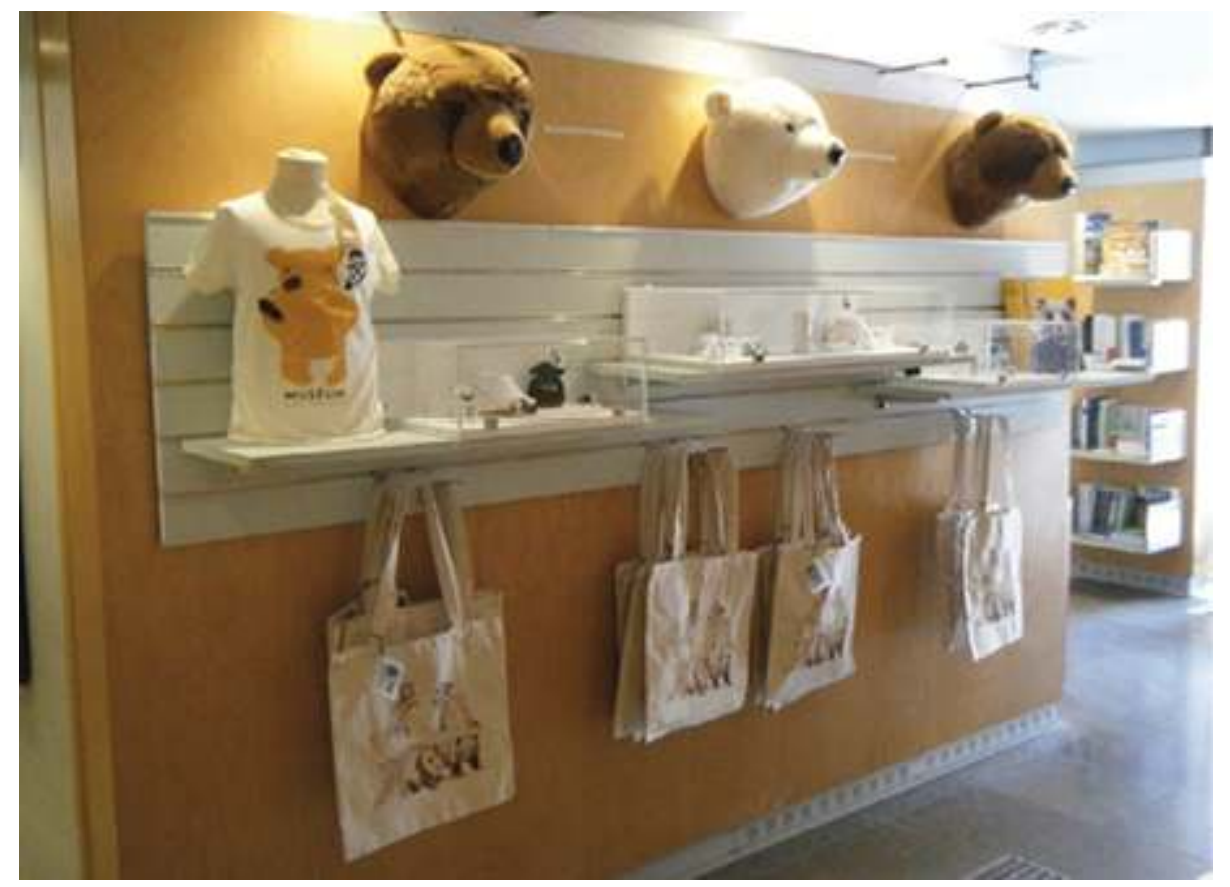

(C) L. Danilo 
Certaines données étant manquantes, notamment pour les analyses nécessitant des éléments financiers, il sera rappelé pour chaque analyse le nombre d'observations contributives, afin de nuancer certains résultats au vu d'un échantillon résolument trop faible. Une nuance générale est également à apporter, un échantillon de 22 observations n'étant pas suffisant, d'un point de vue statistique.

10 Certains résultats étaient attendus, d'autres moins. Y compris dans le cas de résultats attendus, cette analyse apporte une plus-value : elle permet de disposer d'un support tangible, basé sur des résultats factuels, afin de démontrer ou d'appuyer certaines hypothèses, tant auprès de ses tutelles que de ses agents.

\section{Exploration de liens entre variables avec les régressions linéaires}

11 Ces régressions linéaires visent à identifier des variables influençant des facteurs importants pour le fonctionnement d'une boutique, comme le rapport car la boutique en tant que surface commerciale peut être évaluée à l'aune de son rapport. De manière à optimiser celui-ci, quels leviers d'action peuvent être utilisés?

Le rapport chute à mesure que le taux de captation augmente. Ceci est à nuancer au vu du faible nombre d'observations (12) et la valeur du coefficient de corrélation $(r=-0,52)$. Néanmoins, ce résultat peut trouver son explication dans le lien avec d'autres variables : un taux de captation supérieur peut être le signe de produits bon marché attractifs qui font baisser le rapport.

13 En revanche, a priori il n'existe pas de lien entre le panier moyen et le rapport de la boutique : la rentabilité de la boutique n'est pas liée à la quantité d'achats effectués par un client, mais plutôt au nombre de clients ( 9 observations, $r=0,01$ ). Ceci est porteur 
d'informations supplémentaires : dans ce type d'espace, il est préférable de miser davantage sur le marchandisage (mise en scène des produits) que sur la force de vente ou le conseil approfondi.

14 Si le nombre d'articles vendus influence peu le rapport ( 9 observations, $r=-0,30$ ), il semble par contre fortement influencer le panier moyen (10 observations, $r=0,83$ ). Le panier moyen augmente avec le nombre d'articles vendus. Derrière cette assertion somme toute logique, une information importante : ce n'est pas forcément en achetant des produits à prix élevé que le panier augmente, c'est surtout l'achat de davantage de produits à prix moins élevés qui le fait augmenter.

De manière intuitive, le nombre de références semble influer sur le nombre de produits vendus et sur le panier moyen. En effet, ces deux variables augmentent avec l'accroissement du nombre de références proposées (nombre d'articles vendus, $r=0,74,13$ observations ; panier moyen, $r=0,62,15$ observations). Ceci signifie qu'un plus large choix proposé permet d'augmenter les ventes. L'hypothèse suivante peut découler de cette assertion : si l'augmentation du panel proposé permet d'assurer un choix et d'augmenter les ventes, ceci sera peut-être vrai pour chaque sous-ensemble. Ainsi, il serait préférable de s'assurer de la variété dans chaque typologie de produit, plutôt que de multiplier les produits différents avec un faible choix à l'intérieur de chaque gamme.

Si les livres constituent une part importante de l'offre des boutiques d'établissements culturels, ils peuvent être accompagnés d'un cortège d'autres produits, comme ici au muséum d'Histoire naturelle de Toulouse.

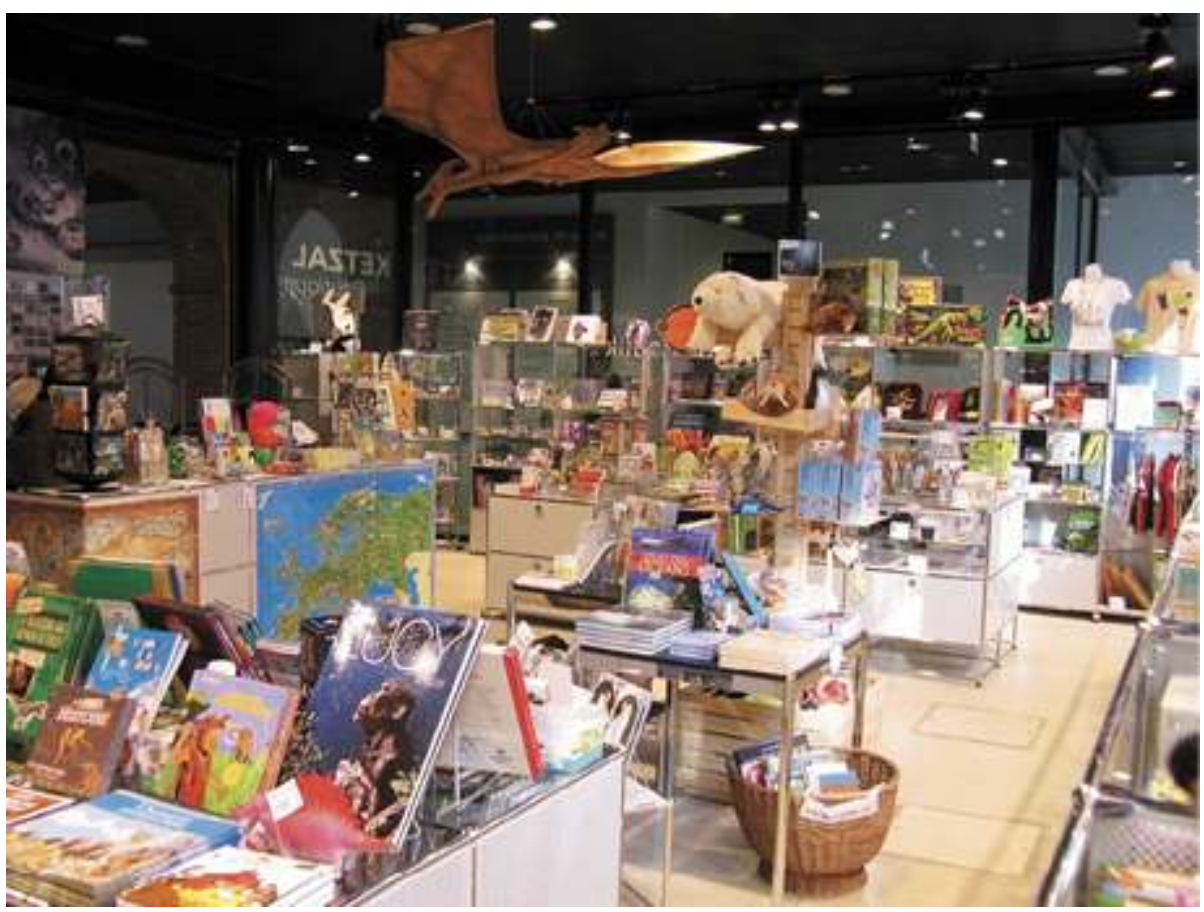

(C) Muséum de Toulouse

Enfin, la question du personnel est centrale au vu des coûts qu'il génère. Aussi, comment gérer au mieux la masse salariale pour la tenue de la boutique ? Les liens entre nombre de personnels et surface de vente paraissent a priori évidents. Ils semblent en effet étroitement liés de manière positive au nombre maximal de personnels présents en boutique, soit aux périodes d'affluence $(\mathrm{r}=0,79)$. Pour les autres périodes, le nombre est 
nettement moins corrélé à la surface $(\mathrm{r}=0,50)$. Ces deux résultats sont soutenus par la totalité de l'échantillon. Il en ressort un point d'amélioration sur l'efficience de la répartition des ressources humaines à la boutique : hors affluence, certains établissements conservent peut-être trop de personnel.

\section{Exploration des influences multiples des variables les unes sur les autres: I'ACP}

17 Les résultats des régressions linéaires précédentes montrent des liens entre variables deux à deux, mais aussi la difficulté d'expliquer une variable seulement avec l'influence d'une autre. L'Analyse en Composantes Principales permet d'avoir une vision plus synthétique des différentes influences au cœur de ce jeu de données.

18 Il ressort de cette analyse que le panier moyen est influencé à la fois par le personnel, par sa formation et par ses missions : un personnel non formé ou peu formé qui cumule plusieurs missions simultanément ou même de manière décalée, influe négativement sur la valeur du panier moyen du client.

19 Par ailleurs, l'implication de différents services comme la médiation ou la communication dans le choix des produits ne représente aucune amélioration de rentabilité. Ceci ne signifie pas que cette option soit considérée comme négative, elle peut influer positivement sur d'autres éléments fondamentaux comme l'image de la boutique et donc du musée ou sur les valeurs à transmettre.

Enfin, de manière contre-intuitive, la présence de la boutique en ligne ou avec une communication développée ne permet pas d'augmenter le taux de captation ou seulement légèrement. Ici, le manque de données ne permet pas de trancher la question. Il se peut que ce résultat soit le signe d'habitudes d'achats en boutiques de musées très liées à la visite et peu préparées à l'avance. Cependant, certains échanges au cours de ces enquêtes montrent ponctuellement l'impact d'une communication ciblée (fêtes de fin d'année), sans que cela ressorte statistiquement. Il n'est pas exclu que les conceptions d'une boutique de musée puissent changer si certaines stratégies sont déployées, mais il est probable que le lien visite/boutique demeure bien plus fort que l'achat extérieur.

\section{Quels produits vendre pour quel potentiel de vente?}

21 La plupart des catégories d'objets sont consensuelles : beaucoup sont présentes dans plus des $3 / 4$ des boutiques sollicitées. Il s'agit des livres, des activités manuelles, des jeux d'expérimentation, des objets à construire, de la papeterie, des bijoux, des sacs, de la vaisselle (mugs), affiches et cartes postales. Viennent ensuite certaines produits présents dans seulement $2 / 3$ des établissements. Il s'agit des DVD, des jeux de société, des figurines, des jouets, des peluches, et du textile, dont la gestion est complexe, notamment à cause des tailles. Les gadgets ne font pas non plus l'unanimité, bien que les résultats de cette question soient partiellement biaisés par des conceptions visiblement différentes de ce qui est ou n'est pas un gadget.

Ces disparités ne semblent pas refléter les différences de collections et donc de publics des établissements, en dehors du cas notable des peluches, toujours présentes dans les 
établissements de culture scientifique qui traitent fréquemment de sujets naturalistes et jamais, du moins dans l'échantillon présenté ici, dans les autres.

Toutes sortes de produits peuvent être "griffés" et porter l'image de l'établissement. Des séries peuvent aussi être envisagées pour stimuler les collections. Ainsi, le Muséum-Aquarium de Nancy vend très facilement ses affiches originales.

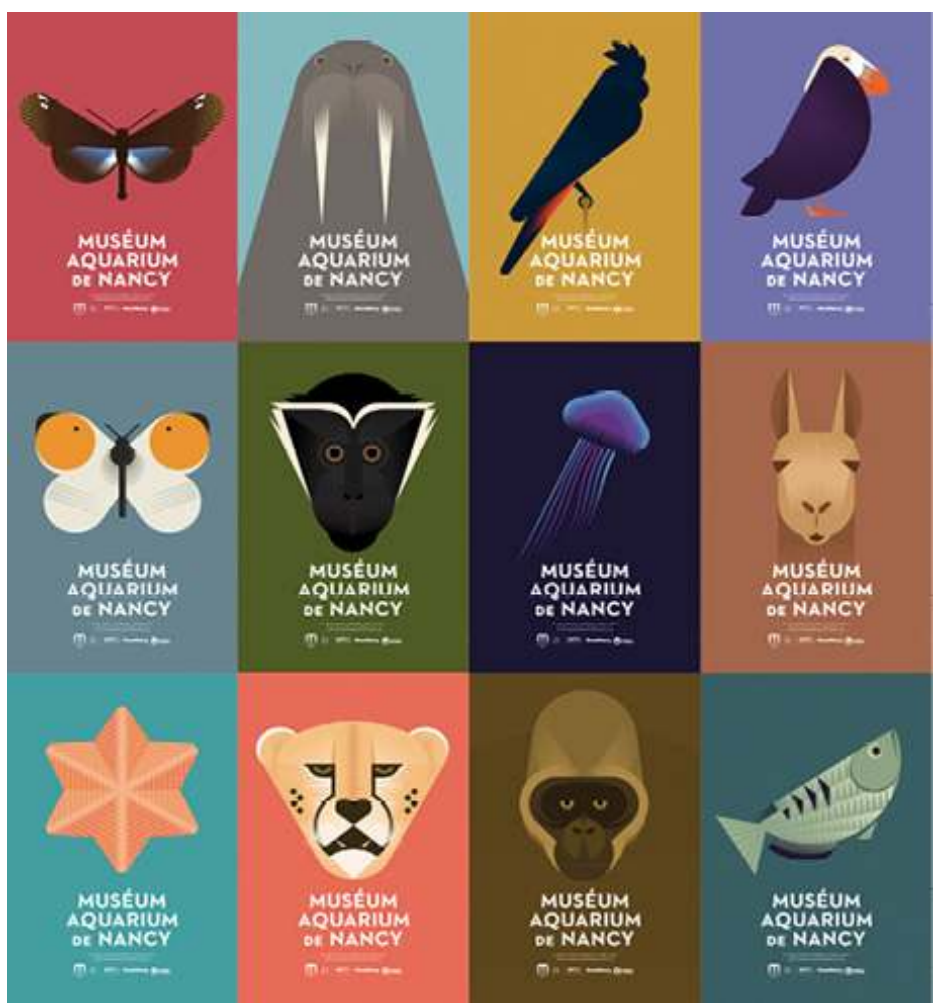

(c) Muséum-Aquarium de Nancy/P. Tytgat

Deux catégories de produits, les "savons et bougies" et les "produits du terroir et spécialités régionales", sont représentées dans moins de la moitié des établissements. Les savons et bougies ne sont pas liés aux propos des musées, ce qui explique qu'un grand nombre s'en dispense, mais ils se vendent plutôt bien. Dans certains cas, ils développent des parfums en lien avec des spécialités régionales, comme le "savon à la mirabelle en Lorraine". Il en va de même pour les spécialités régionales et produits du terroir, mais ceux-ci valorisant un territoire, ils trouvent plus facilement leur place au sein d'une boutique de musée. Le frein principal mentionné à leur égard porte plutôt sur leur gestion, notamment en lien avec leurs dates limites de consommation.

Enfin, seuls deux établissements proposent des cartes cadeaux aux visiteurs. Cela étant, de nombreuses personnes ont manifesté un intérêt pour cette question lors des entretiens.

Les produits qui se vendent le mieux sont principalement les peluches, les produits griffés, la papeterie, les cartes postales et les médailles (monnaie de Paris) portant le visuel de l'établissement. D'une manière générale, les produits qui se vendent le moins sont ceux proposés à un prix supérieur à 30 euros, mais aussi les DVD et $\mathrm{CD}$, les livres spécialisés, les BD, les figurines, le textile, la vaisselle, les affiches coûteuses, la bagagerie, les bijoux. Certains mentionnent aussi les cartes postales comme un produit en perte de vitesse. 
26 Face à cette question des produits les moins vendus, de nombreuses personnes ont précisé que certains de ces produits devaient néanmoins être présents, mais en petites quantités. Il en va ainsi par exemple des livres spécialisés, qui trouveront un public qui saura les apprécier et sera satisfait de les trouver même s'ils ne seront pas vendus fréquemment. Ceci participe de la conception de boutique comme service au public et contribue à l'image de l'établissement. De nombreuses personnes précisent en effet que les visiteurs sont satisfaits de pouvoir trouver en ces lieux des objets et ouvrages, ou un assortiment de produits qui ne sera pas retrouvé ailleurs.

$\mathrm{Au}$ sujet des appréciations des produits les plus ou moins vendus, il faut prendre garde à un biais : beaucoup de boutiques ne réalisent pas de bilan analytique et ces réponses sont fréquemment fournies en lien avec les ressentis des personnes interrogées.

\section{Résultats qualitatifs : que faire ou ne pas faire?}

Être réactif: La réactivité est fréquemment revenue comme un élément clé de la réussite d'une boutique : il faut se renouveler en permanence, pouvoir changer lorsqu'un produit ne fonctionne pas. Et pour cela, il est indispensable de ne pas sur-stocker : il vaut mieux acheter en plus petite quantité, quitte à ne pas bénéficier de prix de gros mais pouvoir tout vendre. En outre, les espaces de stockage sont à prévoir au moins pour partie à proximité immédiate de la boutique, voire dans le mobilier de présentation, afin de pouvoir procéder au réapprovisionnement des rayons en temps utile.

Il est conseillé de ne pas réaliser ses commandes pour toute l'année, mais de les découper en deux, trois, voire quatre commandes, permettant de s'adapter en fonction des ventes et des périodes. Par exemple, les objets proposés seraient différents que l'on soit à Noël ou aux veilles des vacances d'été. Par ailleurs, les produits limités, lorsque leur caractère éphémère est précisé, stimulent les ventes.

Soigner le marchandisage : De l'avis collectif, le marchandisage est extrêmement important. Le temps dévolu au conseil, autant que la formation des agents, ne permettent pas à eux seuls de réaliser les ventes. Les produits, comme dans une exposition, doivent être mis en scène, éclairés et valorisés. La boutique doit être conçue comme un lieu scénographié, où le mobilier et les atmosphères font partie intégrante du projet. Une ambiance peut être donnée à l'espace de vente, tant par les couleurs que le mobilier ou encore la diffusion de musique.

Les produits doivent également être mis en valeur en fonction de leurs caractéristiques : un produit local peut être accompagné du logo de la région, un produit bio du label, coup de cœur du musée... Des offres ponctuelles associant plusieurs objets dans un coffret esthétique peuvent aussi être de nature à stimuler l'achat. 
La boutique peut devenir un lieu scénographié, reflétant une ambiance en lien avec l'établissement. Aux Machines de l'île, à Nantes, la boutique se pare d'un aspect brut et industriel avec tuyaux apparents et mobiliers rappelant les caisses de transports.

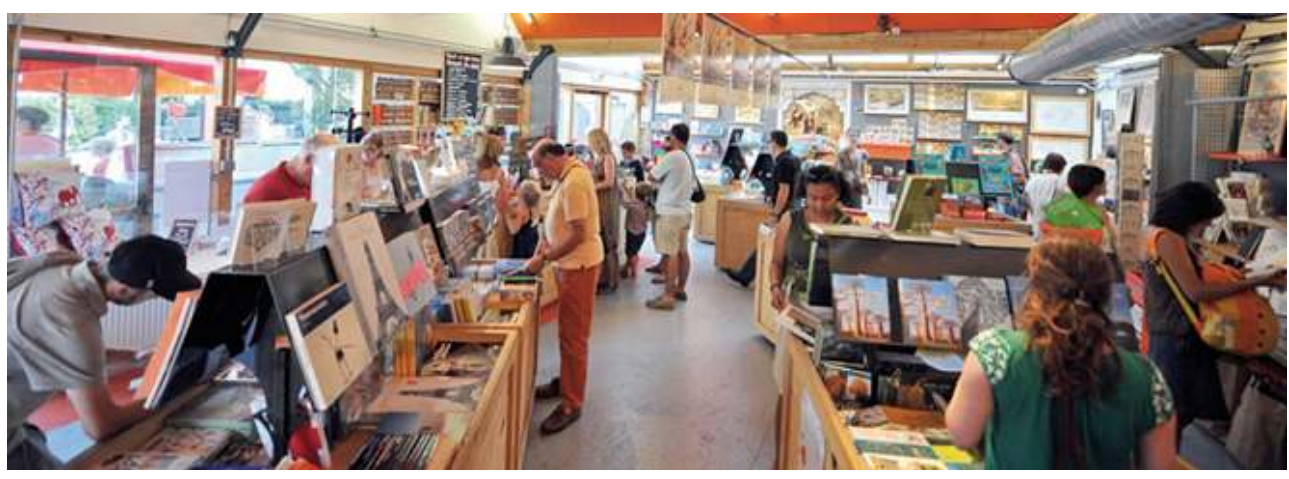

(c) Les Machines de l'île

Autre lieu, autre ambiance : la boutique "Mémoires d'Océans", de l'aquarium de La Rochelle joue sur les bleus et les formes évocatrices des mondes aquatiques.

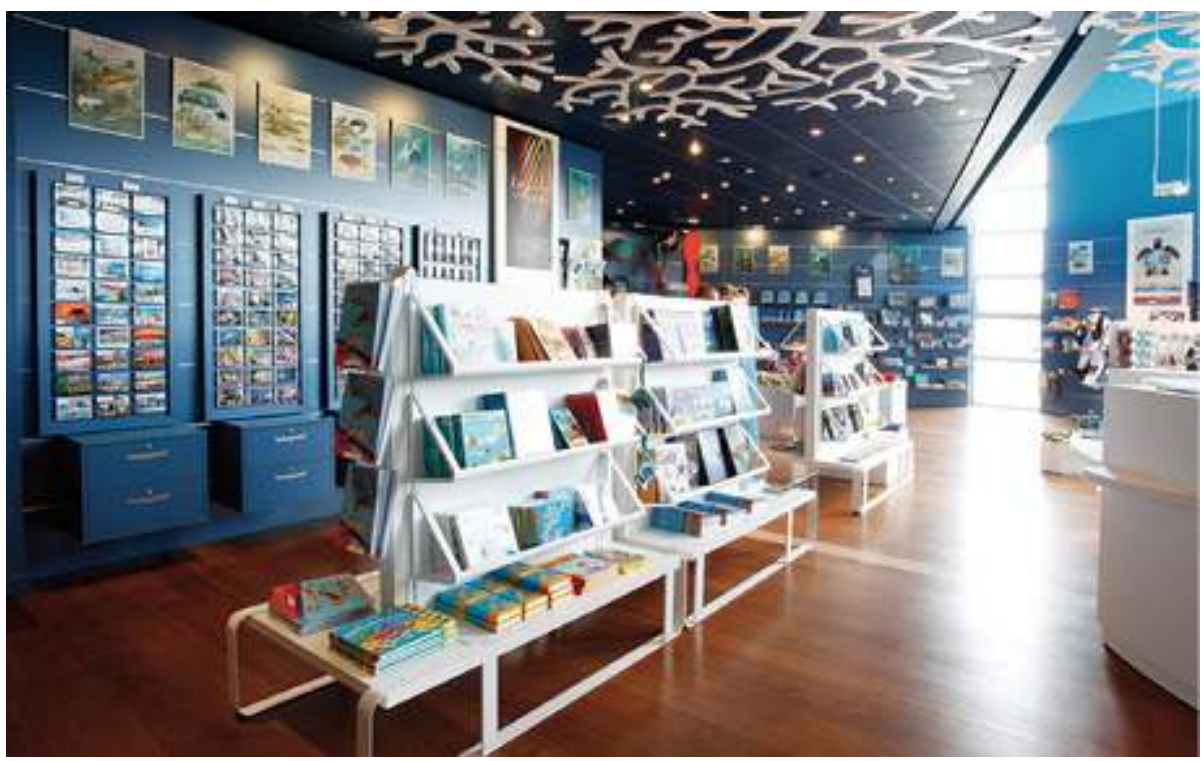

(c) Mémoires d'Océans doivent être votés par l'organe exécutif. Quelques établissements sont parvenus à assouplir leur gestion de stock et leurs ventes. En accord avec les services financiers de leurs collectivités, ils soumettent au vote non plus des prix fixes mais des coefficients minimaux et maximaux pour chaque catégorie de produits. Ainsi, il n'est plus nécessaire de mentionner le prix d'achat et de vente de chaque modèle mais de préciser que pour tel intervalle de prix d'achat de tel produit, il sera vendu au minimum Prix min = prix d'achat $\mathrm{X}$ coefficient minimum, et au maximum Prix max $=$ prix d'achat $\mathrm{X}$ coefficient maximum ${ }^{2}$. À l'issue de l'année, les prix qui ont réellement été pratiqués sont présentés à l'organe exécutif qui entérine et propose éventuellement des modulations pour l'année suivante. être organisées, du "grenier d'expo" qui propose pour un temps limité les produits

La Lettre de l'OCIM, 174 | 2017 
d'anciennes expositions à tarif préférentiel ou encore des ventes privées s'adressant à un public spécifique (détenteur d'une carte, abonné à un réseau social...), des soldes et autres déstockages. Actuellement, de trop nombreuses institutions supportent malheureusement le poids de leur stock invendable. bon fonctionnement de la boutique. Cependant, certains précisent que si le passage obligé est indispensable, il faut se garder de faire un passage obligatoire, souvent mal perçu par les visiteurs dans un établissement culturel. Il faut donc que le parcours fasse passer le visiteur à proximité immédiate de la boutique, sans pour autant l'obliger à cheminer à l'intérieur.

41 Proposer des inédits avec le dépôt-vente : Selon les choix réalisés, la boutique peut comprendre des productions de créateurs. Dans ces cas-là, il peut être intéressant de négocier des collections exclusives avec eux, afin de se démarquer et de proposer des objets uniques. Le dépôt-vente peut être envisagé pour les créations, qui peuvent ou non 
rencontrer leur public et représenter un coût important pour l'établissement si elles ne sont pas vendues. De même, certains libraires acceptent de confier des ouvrages en dépôt-vente, ce qui peut s'avérer utile, notamment pour les ouvrages spécialisés, et assurer une rotation dans les livres proposés.

Passage obligé et passage obligatoire : le flux entrant est symbolisé en jaune, le flux sortant en bleu. Dans le cas A, le passage est obligé : le visiteur voit forcément la boutique et passe devant, il peut décider (ou non) d'y pénétrer. Dans le cas $B$, le passage est obligatoire : le visiteur doit passer par la boutique pour sortir. Cette dernière situation est fréquemment mal vécue.
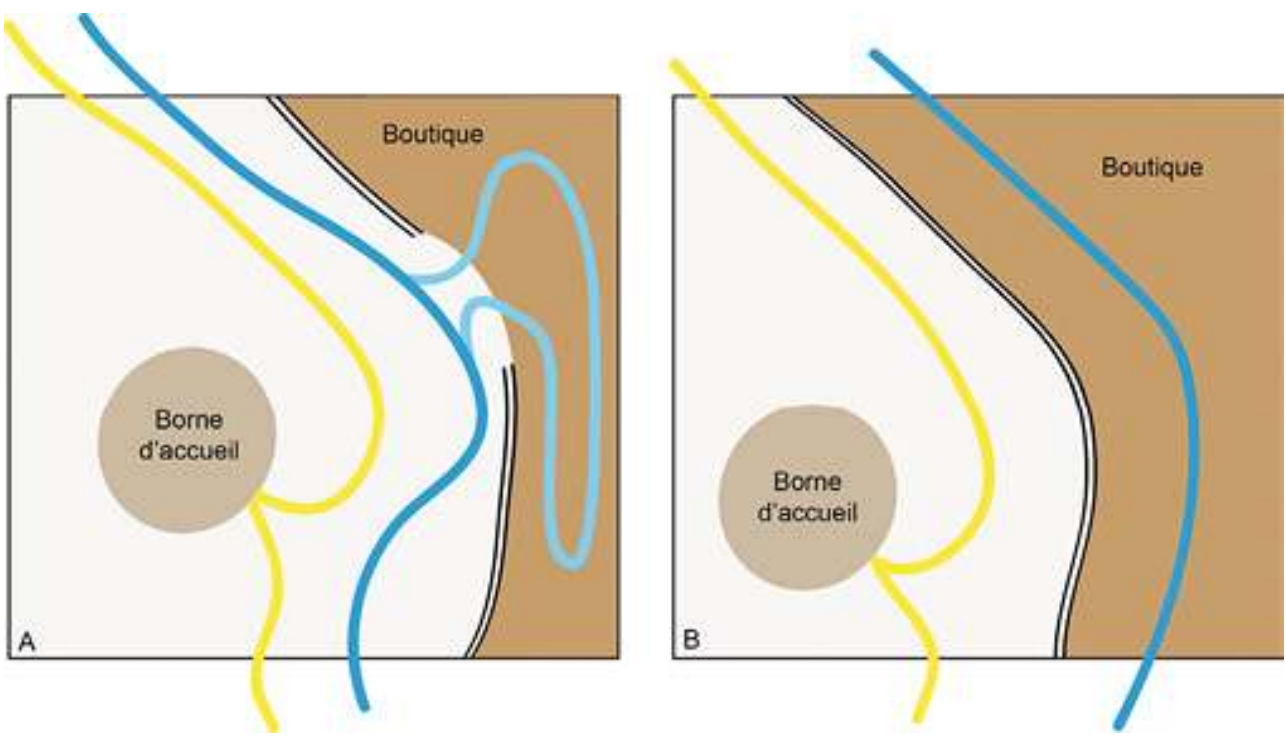

(c) L. Danilo

Entrée de la boutique de la Cité des Sciences et de l'Industrie avant rénovation en 2016 : c'est un passage obligé, mais non obligatoire.

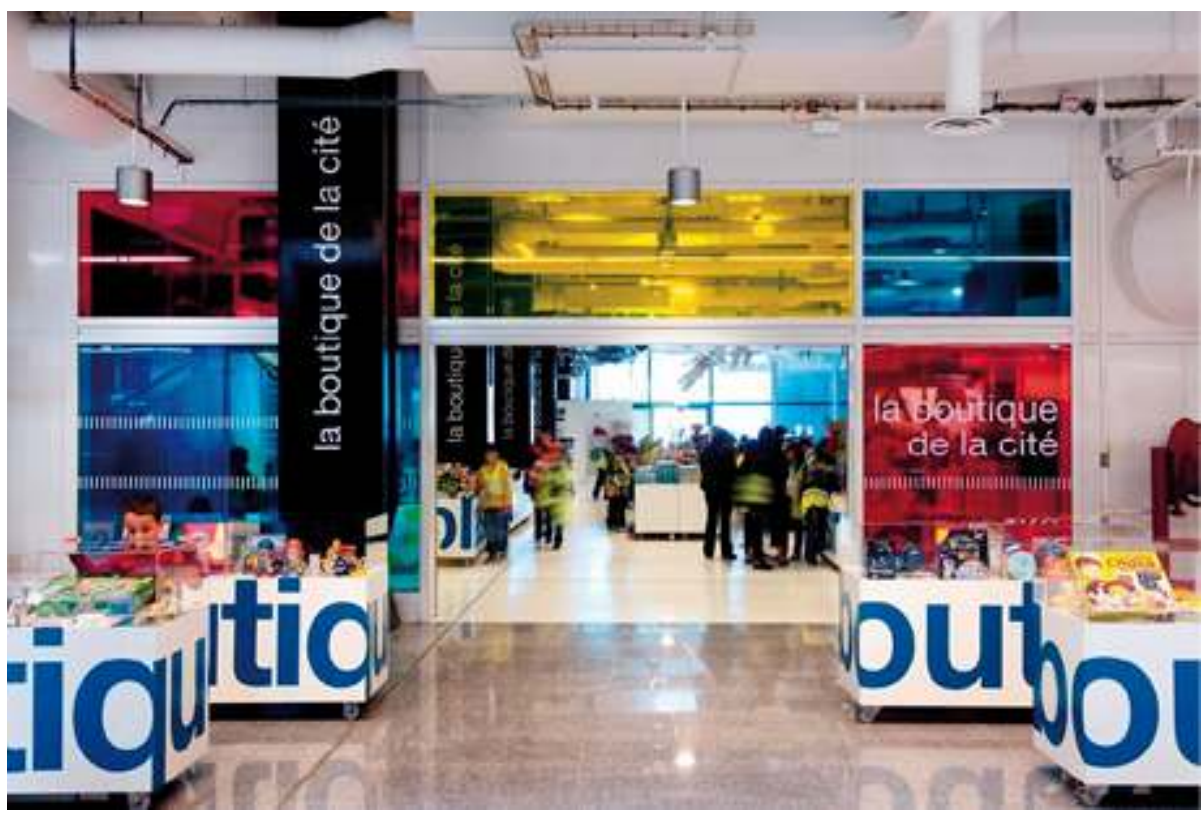

(c) RMN-GP

Présenter des produits réfléchis : Acheter local et favoriser le maintien et la transmission des savoir-faire traditionnels est une préoccupation de nombreux établissements. La 
nécessité de s'inscrire dans une démarche respectueuse de l'environnement est un pilier important pour beaucoup, qui contribue aussi à la transmission de ces valeurs.

Par ailleurs, plus un produit est simple et peu coûteux, plus il sera vendu largement. Aussi, ces produits doivent être encore plus réfléchis car ils représentent davantage leur musée et diffusent son image.

Proposer des horaires décalés : Lorsque c'est possible, décaler légèrement l'ouverture et la fermeture de la boutique par rapport aux horaires du musée permet aux derniers visiteurs de passer par la boutique, voire, en une période de faible affluence, d'échanger sur leur visite.

\section{Conclusion}

Un musée porte en général une vision de transmission éloignée des pratiques mercantiles du monde commercial. L'implantation d'un espace de vente peut ainsi créer des tensions au sein des équipes où certains y voient une perversion des activités du musée ou des impératifs déguisés de rentabilité de la culture. Mais une boutique de musée, si elle peut apporter une manne financière bienvenue aux établissements, doit nécessairement être pensée comme un service au public, une continuité de la visite, et répondre à l'éthique et aux exigences portées par l'établissement.

Schéma synthétique des points les plus importants issus de cette enquête comparative et des analyses auxquelles elle a abouti.

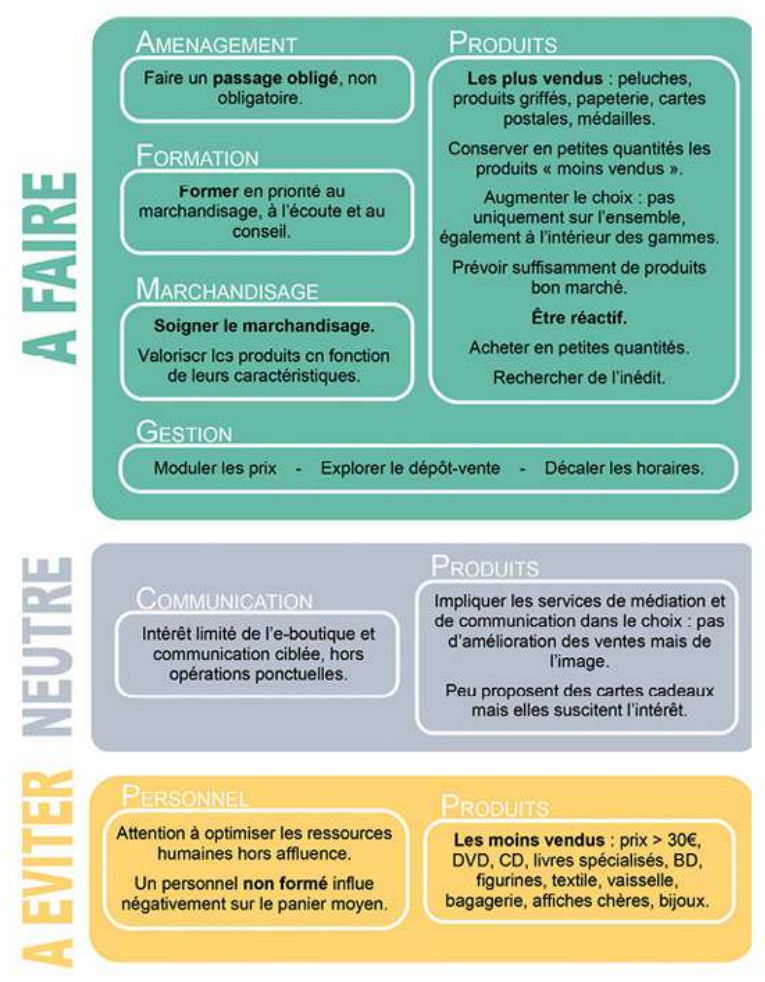

(C) L. Danilo

Ceci n'empêche en rien de s'approprier des outils mercatiques et de s'inspirer des techniques du monde commercial, pour les adapter aux contextes particuliers des 
musées. Ce secteur se renouvèle sans cesse et développe des stratégies inédites. Par exemple, un magasin de Toulouse proposait récemment un moyen original d'attirer l'attention et par là d'éventuels clients : il a installé dans une vitrine donnant sur l'extérieur un compteur connecté à la page Facebook du magasin. Les passants sont invités à "liker" la page, le compteur s'actualisant. Tous les 100 fans, les prix en magasin baissent d'1\%, pour tous, y compris ceux qui n'avaient pas "liké".

\section{BIBLIOGRAPHIE}

Bacher, A.-S. Statuts juridiques des boutiques de musées. Dijon : Ocim, 2000, 59 p.

Cam, J. Boutiques de sites culturels. Guide du gestionnaire. Paris : ODIT France, Ingénierie touristique, 2008,180 p.

Deval, M.-L. Boutiques de musées en France, La Lettre de l'Ocim, n³4, 1994, pp. 8-12.

\section{NOTES}

1. La grande majorité des établissements approchés n'inclut dans son rapport que les dépenses liées aux achats de produits et mobilier de boutique et ne comprend donc pas les fluides ou la masse salariale, leur boutique étant loin de la rentabilité financière. Cependant, une entreprise contactée dans le cadre de cette étude indique qu'elle peut chercher à obtenir une délégation de service public dans un établissement à partir d'un seuil de 120000 visiteurs, qui correspond pour elle au seuil de rentabilité, comprenant cette fois la masse salariale.

2. Par exemple, supposons un prix d'achat de peluches compris entre 5 et 9 euros . Il peut être proposé d'appliquer au minimum un coefficient multiplicateur de 1,5 et au maximum un coefficient de 2 , pour cette catégorie de produits. Pour une peluche à 5 euros il s'ensuivrait un prix minimal de $5^{*} 1,5=7,5$ euros et un prix maximal de $5^{*} 2=10$ euros . Pour une peluche à 9 euros, il s'ensuivrait un prix minimal de $9^{*} 1,5=13,5$ euros et un prix maximal de $9^{*} 2=18$ euros. Avec tous les intermédiaires possibles.

\section{RÉSUMÉS}

Créer et gérer une boutique ne va pas de soi pour un musée : cette analyse statistique et qualitative des résultats d'une enquête comparative menée auprès de plusieurs institutions muséales permet d'évaluer les différentes stratégies mises en place et d'identifier les éléments notamment la notion de service au public et l'inscription du lieu dans la continuité de la visite qui conduisent au bon fonctionnement d'une boutique de musée. 
INDEX

Mots-clés : boutique, musée, visite

\author{
AUTEUR \\ LAURE DANILO \\ conservatrice du patrimoine, responsable du muséum d'Orléans pour la Biodiversité et \\ l'Environnement (Mobe) \\ laure.danilo@orleans-metropole.fr
}

